\title{
Optimal pain management for radical prostatectomy surgery: what is the evidence?
}

\author{
Grish P. Joshi ${ }^{*}$, Thomas Jaschinski ${ }^{2}$, Francis Bonnet ${ }^{3}$, Henrik Kehlet ${ }^{4}$ and on behalf of the PROSPECT collaboration
}

\begin{abstract}
Background: Increase in the diagnosis of prostate cancer has increased the incidence of radical prostatectomy. However, the literature assessing pain therapy for this procedure has not been systematically evaluated. Thus, optimal pain therapy for patients undergoing radical prostatectomy remains controversial.

Methods: Medline, Embase, and Cochrane Central Register of Controlled Trials were searched for studies assessing the effects of analgesic and anesthetic interventions on pain after radical prostatectomy. All searches were conducted in October 2012 and updated in June 2015.

Results: Most treatments studied improved pain relief and/or reduced opioid requirements. However, there were significant differences in the study designs and the variables evaluated, precluding quantitative analysis and consensus recommendations.

Conclusions: This systematic review reveals that there is a lack of evidence to develop an optimal pain management protocol in patients undergoing radical prostatectomy. Most studies assessed unimodal analgesic approaches rather than a multimodal technique. There is a need for more procedure-specific studies comparing pain and analgesic requirements for open and minimally invasive surgical procedures. Finally, while we wait for appropriate procedure specific evidence from publication of adequate studies assessing optimal pain management after radical prostatectomy, we propose a basic analgesic guideline.
\end{abstract}

Keywords: Radical prostatectomy, Postoperative, Pain, Multimodal analgesia

\section{Background}

Prostate cancer is the most common cancer in men, with more than 240.000 patients newly diagnosed per year in the United States alone [1]. Radical prostatectomy remains one of the key techniques to treat prostate cancer [2], and the incidence of surgery has risen with improved prostate-specific antigen screening programmes [3, 4].

Optimal pain management is known to influence postoperative recovery [5], but patients undergoing open radical prostatectomy typically experience moderate dynamic pain in the immediate postoperative days [6]. Robot-assisted and laparoscopic surgery may be associated with decreased

\footnotetext{
*Correspondence: girish.joshi@utsouthwestern.edu

'Department of Anesthesiology and Pain Management, University of Texas Southwestern Medical School, 5323 Harry Hines Blvd, Dallas, TX 75390-9068, USA

Full list of author information is available at the end of the article
}

pain levels as opposed to open surgery [6], but even here, abdominal and incisional pain are prominent sources of moderate dynamic pain scores $[7,8]$.

The literature assessing the efficacy of various analgesic drugs and techniques in patients undergoing radical prostatectomy has not been systematically evaluated. Consequently, optimal pain therapy for patients undergoing radical prostatectomy remains to be defined.

The aim of the present systematic review is to evaluate the available literature on the management of pain after radical prostatectomy. Postoperative pain outcomes (e.g., pain scores and supplemental analgesic requirements) are the primary focus, but other recovery outcomes, including adverse effects, are also assessed where reported, and the limitations of the data are reviewed. This systematic review will also be used to determine the knowledge gaps, which will guide future research. In addition, this 
review can serve as a starting point for developing recommendations for clinical decision-making in the management of pain after radical prostatectomy surgery.

\section{Methods}

\section{Systematic literature search}

Medline, Embase, and the Cochrane Central Register of Controlled Trials were searched for studies comparing analgesic and anesthetic interventions in patients undergoing radical prostatectomy according to the Preferred Reporting Items for Systematic reviews and MetaAnalyses (PRISMA) guidelines [9]. All searches were conducted in October 2012 without restriction to the publication date by using a combination of text words and data-base specific controlled terms related to prostatectomy, analgesia and pain assessment. We also manually retrieved publications referred in studies identified by our preceding search. The search was updated in June 2015.

\section{Study inclusion and selection}

The selection process was performed in a two-step procedure. First, two reviewers selected studies independently by screening the titles and abstracts according to predefined inclusion criteria: randomized controlled trials (RCTs) published as full-text in English assessing analgesic, anesthetic and surgical techniques affecting postoperative pain in patients undergoing radical prostatectomy. In studies with mixed surgical procedures there had to be a defined prostatectomy subgroup. After retrieving potential relevant studies, full-texts were checked against the inclusion criteria once again. Any disagreements were resolved by consensus. In the case of insolvable discrepancies, a third reviewer was involved in the discussion.

\section{Quality assessment and outcome analysis}

For the critical appraisal of included studies we used the Cochrane Collaboration's tool for assessing the risk of bias [10]. The data extraction tables summarize pain scores, supplementary analgesic use and time to first analgesic requirement. It was assumed that the postoperative pain scores were assessed at rest, unless otherwise specified in the study report. Studies were stratified according to the regimen (analgesic, anesthetic and operative), mode of delivery (systematic or local) and class of agent. The assessment of the risk of bias and data extraction were conducted by one author and checked by a second author. Any disagreements were resolved by discussion or by consultation of a third reviewer. Quantitative metaanalyses were not performed, owing to the limited number of included studies with homogenous designs reporting similar outcome measures.

\section{Results}

\section{Study selection process}

In the search until October 2012, 38 studies met the inclusion criteria (Fig. 1), of which, an open approach was performed in 34 studies [11-43], a laparoscopic approach was performed in 1 study [44], and a robotic-assisted laparoscopic approach was performed in 3 studies [45-47]. Due to insufficient reporting the surgical approach was unclear in one study [48].

\section{Risk of bias in included studies}

The quality of all included studies was moderate to poor and most studies had similar flaws (Table 1). For the qualitative analysis the trials were assigned to 2 broad groups: pharmacological techniques and anesthetic techniques. There were no studies that compared or utilized multimodal pain interventions.

\section{Pharmacological interventions}

The trials assessing analgesic interventions were grouped into conventional analgesics (non-selective non-steroidal anti-inflammatory drugs (NSAIDs), cyclooxygenase (COX) 2-selective inhibitors, lidocaine, and opioids); adjunct drugs with analgesic activity ( $\alpha 2$ agonists, $\alpha 2 \delta$ ligands [gabapentin and pregabalin], muscarinic receptor antagonists and N-methyl-D-aspartic acid [NMDA] antagonists [magnesium and ketamine]) (Table 2); and regional anesthesia techniques generally showed that these pharmacological approaches were useful (Table 3). Four studies compared epidural analgesia with systemic analgesia, of which two showed a reduction in pain scores (Table 3). Two studies evaluated intrathecal opioids with or without clonidine (Table 3). Both showed improved pain relief, but increased frequency of pruritus was reported in one study.

\section{Surgical techniques}

Although a minimally invasive approach for radical prostatectomy has been rapidly adopted in clinical practice $[3,4]$, there are only 4 RCTs assessing pain management. Moreover, between October 2012 and June 2015, only 2 additional RCT have been published assessing pain control using a robotic approach [49, 50]. These studies focused on adjunct techniques (i.e., penile block to improve bladder catheter tolerance [49] and intravesical ropivacaine [50]) and both did not result in any improvement in pain control.

\section{Anesthetic techniques}

Three studies investigating the use of regional anesthesia, including combined procedures with general anesthesia, showed a reduction of analgesic supplemental use with regional anesthesia (Table 4). However, the differences between groups with regard to pain scores were inconclusive. 


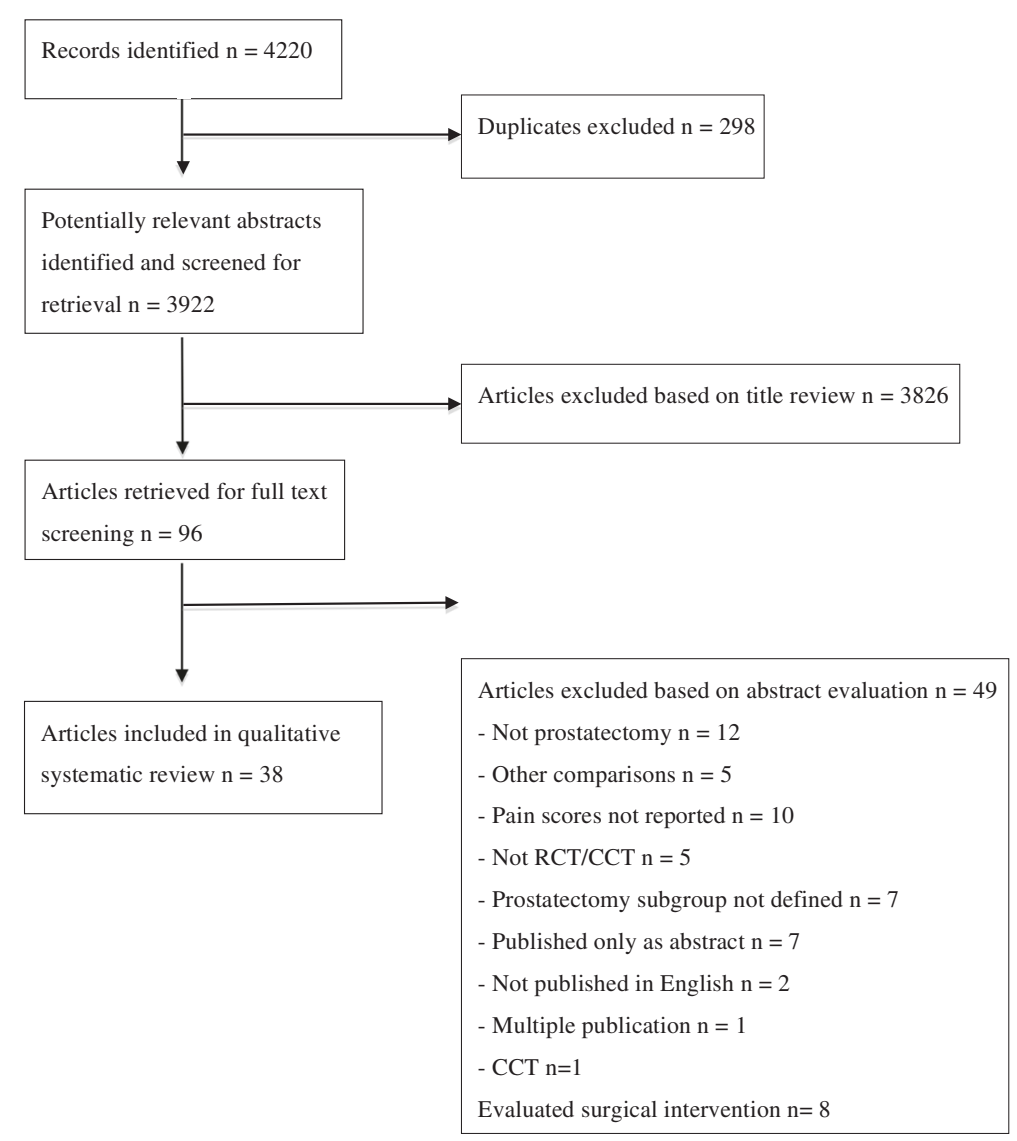

Fig. 1 PRISMA diagram showing identification of included studies

Two studies compared spinal anesthesia with general anesthesia. Patients receiving spinal anesthesia had significantly shorter durations of surgery, reduced blood loss and lower pain scores on the day of surgery than patients receiving general anesthesia.

\section{Discussion}

This systematic review reveals that there is a significant lack of evidence to develop an optimal pain management protocol in patients undergoing radical prostatectomy. Most studies evaluating pain management after radical prostatectomy surgery assessed unimodal analgesic approaches [11-48]. The optimal dose or timing of administration of analgesic agents could not always be determined. Although it is generally accepted that minimal access surgery for radical prostatectomy reduces postoperative pain, it is poorly studied.

Pain after laparoscopic/robotic prostatectomy is generally mild-to-moderate [7]. A recent observational, prospective cohort study that included a limited number of opioid-naïve patients reported that pain after robotic radical prostatectomy was adequately controlled primarily with NSAIDs and opioids [47]. Because opioids may delay recovery and increase the length of hospital stay
[51], due to opioid-related adverse effects such as nausea, vomiting and prolonged postoperative ileus [52], nonopioid analgesics and/or regional analgesic techniques should be used as primary analgesics, and supplemented with opioids, only if necessary.

While we wait for appropriate procedure specific evidence for optimal pain management after minimally invasive radical prostatectomy, a basic analgesic technique, used in observational trials [7], could include a combination of acetaminophen (paracetamol) and NSAID or COX-2 selective inhibitor along with wound infiltration of the trocar sites [5]. The choice between a traditional NSAID and COX-2 selective inhibitors should depend upon assessment of individual patient risks. Non-selective NSAIDs can increase the potential risk of bleeding [53] in contrast to COX-2 selective inhibitors. However, a recent randomized, placebo-controlled, double-blind trial in patients undergoing open prostatectomy reported that while parecoxib reduced opioid use and opioid-related side effects, blood loss at $24 \mathrm{~h}$ after surgery was significantly higher in comparison to the placebo group, corresponding to a $1 \mathrm{~g} / \mathrm{dL}$ difference in hemoglobin [54].

For patients undergoing open prostatectomy under spinal anesthesia, intrathecal morphine may be an appropriate 
Table 1 Methodological quality summary and level of evidence (LoE)

\begin{tabular}{|c|c|c|c|c|c|c|c|}
\hline Study & $\begin{array}{l}\text { Generation of } \\
\text { allocation sequence }\end{array}$ & $\begin{array}{l}\text { Allocation } \\
\text { concealment }\end{array}$ & $\begin{array}{l}\text { Blinding of } \\
\text { participants and } \\
\text { personnel }\end{array}$ & $\begin{array}{l}\text { Blinding of } \\
\text { outcome } \\
\text { assessment }\end{array}$ & $\begin{array}{l}\text { Incomplete } \\
\text { outcome data }\end{array}$ & $\begin{array}{l}\text { Selective outcome } \\
\text { reporting }\end{array}$ & LoE \\
\hline Allaire 1992 [11] & O & O & - & - & + & $\mathrm{O}$ & 2 \\
\hline Andrieu 2009 [12] & $\mathrm{O}$ & $\mathrm{O}$ & - & - & $\mathrm{O}$ & - & 2 \\
\hline Aribogan 2002 [13] & O & O & - & + & O & - & 2 \\
\hline Bilgin 2011 [14] & + & $\mathrm{O}$ & + & + & $\mathrm{O}$ & - & 1 \\
\hline Borazan 2010 [15] & + & O & + & O & O & O & 1 \\
\hline Brown 2004 [16] & + & $\mathrm{O}$ & + & $\mathrm{O}$ & + & $\mathrm{O}$ & 1 \\
\hline Chelly 2011 [17] & + & $\mathrm{O}$ & + & + & + & - & 1 \\
\hline Fant 2011 [18] & + & O & + & O & + & $\mathrm{O}$ & 1 \\
\hline Gaitini 1996 [19] & $\mathrm{O}$ & $\mathrm{O}$ & - & - & $\mathrm{O}$ & - & \\
\hline Gottschalk 1998 [20] & $\mathrm{O}$ & $\mathrm{O}$ & + & + & $\mathrm{O}$ & $\mathrm{O}$ & 1 \\
\hline Groudine 1998 [21] & + & $\mathrm{O}$ & + & + & + & $\mathrm{O}$ & 1 \\
\hline Gupta 2006 [22] & + & $\mathrm{O}$ & + & + & + & - & 1 \\
\hline Habib 2008 [23] & O & O & + & + & + & O & 1 \\
\hline Habib 2009 [24] & + & + & + & + & + & - & 1 \\
\hline Haythornthwaite 1998 [25] & O & O & - & + & O & $\mathrm{O}$ & \\
\hline Heid 2007 [26] & + & $\mathrm{O}$ & + & + & $\mathrm{O}$ & - & 1 \\
\hline Hohwu 2006 [27] & $\mathrm{O}$ & + & - & - & + & $\mathrm{O}$ & 1 \\
\hline Hong 2011 [28] & + & $\mathrm{O}$ & + & + & + & O & 1 \\
\hline Huang 2001 [29] & + & $\mathrm{O}$ & + & + & $\mathrm{O}$ & $\mathrm{O}$ & 1 \\
\hline Katz 2004 [30] & + & + & + & + & + & $\mathrm{O}$ & 1 \\
\hline Liu 1995 [31] & $\mathrm{O}$ & $\mathrm{O}$ & + & + & $\mathrm{O}$ & $\mathrm{O}$ & 1 \\
\hline Mayson 2000 [32] & + & $\mathrm{O}$ & + & + & + & $\mathrm{O}$ & 1 \\
\hline Mazaris 2008 [33] & $\mathrm{O}$ & $\mathrm{O}$ & & & $\mathrm{O}$ & $\mathrm{O}$ & 2 \\
\hline Ormiston 1981 [34] & $\mathrm{O}$ & $\mathrm{O}$ & + & $\mathrm{O}$ & + & - & 1 \\
\hline Salonia 2006 [36] & + & $\mathrm{O}$ & - & - & $\mathrm{O}$ & $\mathrm{O}$ & 2 \\
\hline Shir 1994 [37] & $\mathrm{O}$ & $\mathrm{O}$ & - & + & $\mathrm{O}$ & $\mathrm{O}$ & 2 \\
\hline Snijdelaar 2004a [38] & + & $\mathrm{O}$ & + & O & + & $\mathrm{O}$ & 1 \\
\hline Snijdelaar 2004b [39] & + & O & + & + & + & - & 1 \\
\hline Tauzin-Fin 2006 [40] & + & $\mathrm{O}$ & + & + & $\mathrm{O}$ & $\mathrm{O}$ & 1 \\
\hline Tauzin-Fin 2007 [41] & + & O & + & + & + & O & 1 \\
\hline Tauzin-Fin 2009 [42] & + & O & + & + & + & O & 1 \\
\hline Wu 2005 [43] & + & $\mathrm{O}$ & + & + & $\mathrm{O}$ & $\mathrm{O}$ & 1 \\
\hline Lauwick 2009 [44] & + & + & + & $\mathrm{O}$ & $\mathrm{O}$ & $\mathrm{O}$ & 1 \\
\hline Hong 2009 [45] & + & $\mathrm{O}$ & - & + & + & - & 1 \\
\hline Lee 2011 [46] & O & $\mathrm{O}$ & - & + & O & O & \\
\hline Lukasewycz 2010 [47] & + & O & + & + & + & - & 1 \\
\hline Larijani 2004 [48] & O & $\mathrm{O}$ & + & $\mathrm{O}$ & O & - & \\
\hline Weinberg 2014 [49] & + & $\mathrm{O}$ & + & + & + & $\mathrm{O}$ & 1 \\
\hline Fuller 2013 [50] & + & + & $\mathrm{O}$ & + & + & + & 1 \\
\hline Dirkmann 2015 [55] & + & + & + & + & + & + & 1 \\
\hline Nuri-Deniz 2013 [56] & + & O & - & + & + & + & 1 \\
\hline
\end{tabular}


Table 1 Methodological quality summary and level of evidence (LoE) (Continued)

\begin{tabular}{|c|c|c|c|c|c|c|c|}
\hline Ozbek 2013 [57] & + & - & + & + & $\mathrm{O}$ & + & 1 \\
\hline Elkassabany 2013 [59] & + & + & + & + & + & + & 1 \\
\hline Kristensen 2013 [61] & + & + & + & + & + & + & 1 \\
\hline Deniz 2012 [62] & + & - & - & 0 & + & $\mathrm{O}$ & 1 \\
\hline
\end{tabular}

'+' low risk of bias; '-' high risk of bias; 'O' unclear risk of bias

Table 2 Summary of key results from included studies evaluating pharmacological interventions in patients undergoing radical prostatectomy

\begin{tabular}{|c|c|c|c|c|}
\hline Reference & Intervention studied & Pain scores & $\begin{array}{l}\text { Supplementary } \\
\text { analgesia }\end{array}$ & $\begin{array}{l}\text { Time to first } \\
\text { analgesic request }\end{array}$ \\
\hline \multicolumn{5}{|c|}{ Non-steroidal Anti-Inflammatory Drugs (NSAIDs) } \\
\hline Mazaris 2008 [33] & lornoxicam versus paracetamol & $\downarrow$ at rest & NS & - \\
\hline Ormiston 1981 [34] & aspirin versus tiaprofenic acid & NS at rest & - & - \\
\hline Bilgin $2011[14]$ & Diclofenac, IM versus placebo & $\downarrow \downarrow$ at rest & $\downarrow \downarrow$ & - \\
\hline Dirkmann 2015 [55] & Parecoxib versus placebo & $\downarrow \downarrow$ & $\downarrow \downarrow$ & - \\
\hline \multicolumn{5}{|c|}{ Cyclo-oxygenase-2 Selective Inhibitors } \\
\hline Huang 2001 [29] & rofecoxib versus placebo & NS at rest & NS & - \\
\hline Chelly 2011 [17] & celecoxib versus placebo & $\downarrow$ at rest & $\downarrow$ & - \\
\hline \multicolumn{5}{|l|}{ Lidocaine Infusion } \\
\hline Groudine 1998 [21] & lidocaine versus placebo & $\downarrow \downarrow$ at rest & NS & - \\
\hline Lauwick 2009 [44] & lidocaine versus placebo* & NS at rest & $\downarrow$ & - \\
\hline \multicolumn{5}{|l|}{ Opioids } \\
\hline Larijani 2004 [48] & morphine versus placebo & $\downarrow \downarrow$ at rest & - & NS \\
\hline Gaitini 1996 [19] & buprenorphine versus morphine & NS at rest & - & - \\
\hline \multicolumn{5}{|l|}{ Topical Administration } \\
\hline Habib 2008 [24] & nicotine versus placebo & NS at rest and on coughing & $\downarrow \downarrow$ & - \\
\hline Habib 2009 [23] & lidocaine versus placebo & $\downarrow \downarrow$ at rest and on coughing & NS & - \\
\hline \multicolumn{5}{|l|}{ Analgesic Adjuncts } \\
\hline \multicolumn{5}{|l|}{ a2 agonists } \\
\hline Mayson 2000 [32] & clonidine versus placebo & NS at rest and on coughing & NS & - \\
\hline \multicolumn{5}{|c|}{ Muscarinic receptor antagonists } \\
\hline Tauzin-Fin 2007 [40] & oxybutynin versus placebo & $\downarrow \downarrow$ at rest & $\downarrow \downarrow$ & - \\
\hline Lukasewycz 2010 [47] & belladonna and opium versus placebo* & $\downarrow$ at rest NS on movement & NS & \\
\hline \multicolumn{5}{|c|}{ N-methyl-D-aspartic acid (NMDA) antagonists } \\
\hline Tauzin-Fin 2006 [40] & magnesium versus placebo & NS at rest & $\downarrow \downarrow$ & NS \\
\hline Katz 2004 [30] & ketamine versus placebo & NS at rest & NS & - \\
\hline Snijdelaar 2004a [38] & ketamine versus placebo & $\downarrow \downarrow$ at rest NS on movement & $\downarrow \downarrow$ & - \\
\hline Snijdelaar 2004b [39] & amantadine versus placebo & NS at rest & $\downarrow \downarrow$ & - \\
\hline \multicolumn{5}{|l|}{ Melatonin } \\
\hline Borazan 2010 [15] & melatonin versus placebo & $\downarrow \downarrow$ at rest & $\downarrow \downarrow$ & - \\
\hline \multicolumn{5}{|l|}{ Gabapentin } \\
\hline Deniz 2012 [62] & Gabapentin versus placebo & $\downarrow \downarrow$ at rest for $2 \mathrm{~h}$ postop & NS & - \\
\hline
\end{tabular}


Table 3 Summary of key results from included studies evaluating local/regional analgesia techniques in patients undergoing radical prostatectomy ( ${ }^{a}$ indicates laparoscopic or robotic approach)

\begin{tabular}{|c|c|c|c|c|}
\hline Reference & Intervention studied & Pain scores & $\begin{array}{l}\text { Supplementary } \\
\text { analgesia }\end{array}$ & $\begin{array}{l}\text { Time } \\
\text { to first } \\
\text { analgesic } \\
\text { request }\end{array}$ \\
\hline \multicolumn{5}{|c|}{ Epidural analgesia versus systemic analgesia } \\
\hline Allaire 1992 [11] & Epidural fentanyl versus morphine & $\downarrow \downarrow$ at rest & - & - \\
\hline Gupta 2004 [22] & $\begin{array}{l}\text { Epidural ropivacaine, fentanyl and } \\
\text { adrenaline plus placebo via IV-PCA } \\
\text { versus epidural placebo and } \\
\text { morphine via IV-PCA }\end{array}$ & $\downarrow \downarrow$ at rest and on coughing & - & - \\
\hline Hohwü 2006 [27] & $\begin{array}{l}\text { Epidural ropivacaine versus bupivacaine } \\
\text { infiltration + oral oxycodone }\end{array}$ & NS & - & - \\
\hline Liu 1995 [31] & $\begin{array}{l}\text { Epidural hydromorphone versus } \\
\text { hydromorphone via IV-PCA }\end{array}$ & NS at rest and on coughing & - & - \\
\hline
\end{tabular}

Perioperative epidural analgesia versus

postoperative epidural analgesia

Gottschalk 1998 [20] Preemptive epidural fentanyl (4 $\mu \mathrm{g} / \mathrm{kg})$ versus preemptive epidural bupivacaine $(5 \mathrm{mg} / \mathrm{ml})$ and postoperative morphine versus postoperative morphine and bupivacaine. All patients received postoperative epidural morphine $(0.1 \mathrm{mg} / \mathrm{ml})$ and bupivacaine $(0.5 \mathrm{mg} / \mathrm{mL})$

Hong 2011 [28] Epidural ropivacaine versus epidural ropivacaine $(3 \mathrm{mg} / \mathrm{ml})$ plus sufentanil

$(1 \mathrm{\mu g} / \mathrm{ml})$ versus epidural placebo

Components of epidural analgesia
Aribogan 2003 [13] Epidural combination of tramadol and bupivacaine versus tramadol only versus bupivacaine alone

Heid 2007 [26] Epidural ropivacaine versus bupivacaine Epidural analgesia versus local infiltration analgesia

$\begin{array}{ll}\text { Fant } 2011 \text { [18] } & \text { Epidural ropivacaine and fentanyl } \\ & \text { versus ropivacaine via intra-abdominal } \\ & \text { catheter }\end{array}$

Intrathecal Opioids

Andrieu 2009 [12]
Brown 2004 [16] Intrathecal morphine $0.2 \mathrm{mg}$ and clonidine $75 \mu \mathrm{g}$

Nuri Deniz 2013 [56] Intrathecal morphine 0.2 mg

Wound infiltration versus placebo

$$
\begin{array}{ll}
\text { Wu 2005 [43] } & \text { Subfascial bupivacaine versus placebo } \\
\text { Kristensen 2013 [61] } & \text { subfascial bupivacaine versus placebo } \\
\text { Elkassabany 2013 [59] } & \text { TAP block versus placebo }
\end{array}
$$

Penile nerve block

$$
\begin{aligned}
& \text { Weinberg } 2014 \text { [49] } \begin{array}{l}
\text { Dorsal penile nerve block with } \\
\text { bupivacaine vs. placebo }
\end{array}
\end{aligned}
$$

$\downarrow \downarrow$ at rest

Intrathecal morphine $4 \mu \mathrm{g} / \mathrm{kg}$ versus morphine $4 \mu \mathrm{g} / \mathrm{kg}$ plus clonidine 1 $\mu \mathrm{g} / \mathrm{kg}$ versus placebo

$\downarrow \downarrow$ at rest and on movement in both treatment groups for $18 \mathrm{~h}$. Clonidine extended duration by $6 \mathrm{~h}$. $\downarrow \downarrow$

$\downarrow \downarrow$

NS at rest and on movement

NS

$\downarrow \downarrow$

NS

$\downarrow \downarrow$ on movement

NS on movement $\downarrow \downarrow$

$\downarrow$

$\downarrow \downarrow$

NS

NS

$\downarrow \downarrow$

NS

$\downarrow \downarrow$

$\downarrow \downarrow$ anaesthesia versus placebo under remifentanil-based anaesthesia magnesium under remifentanil-based
NS 
Table 3 Summary of key results from included studies evaluating local/regional analgesia techniques in patients undergoing radical

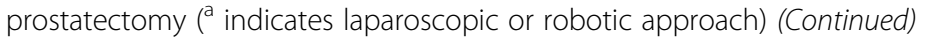

\begin{tabular}{|c|c|c|c|c|}
\hline & $\begin{array}{l}\text { anaesthesia } \\
\text { versus placebo under desflurane-based } \\
\text { anaesthesia }^{\mathrm{a}}\end{array}$ & & & \\
\hline Tauzin-Fin 2009 [42] & $\begin{array}{l}\text { Infiltration of ropivacaine plus } \\
\text { magnesium versus } \\
\text { infiltration of ropivacaine plus } \\
\text { magnesium, IV }\end{array}$ & NS at rest & $\downarrow \downarrow$ & $\downarrow \downarrow$ \\
\hline
\end{tabular}

NA not analyzed, NS no significant difference between groups

- not reported

$\downarrow$, decreased at a minority (50\% or less) of time points measured

$\downarrow \downarrow$, decreased at more than $50 \%$ of time points measured

alternative, assuming that proper precautions are taken for prevention of the morphine-related complications such as nausea and vomiting, pruritus, and respiratory depression. This is also supported by two recent studies reporting reduced intravenous opioid requirements after intrathecal morphine (150-200 $\mu \mathrm{g})$, with a consequent decrease in the incidence of nausea and vomiting $[55,56]$. However, there is a lack of data supporting superiority of epidural analgesia for this surgical procedure; two studies in this systematic review reported benefit from epidural analgesia [11, 22], while two studies found no benefit of epidural analgesia over systemic analgesia [27, 31]. A recent study published after the completion of the systematic review reported that epidural analgesia increased by one day, the length of hospital stay and recommended its avoidance [57].

Two recent studies published after the deadline for inclusion in this systematic review, report controversial results concerning the analgesic effect of the transversus abdominis plane (TAP) blocks included in multimodal protocols $[58,59]$. One placebo-controlled study published after the deadline of this systematic review reported that postoperative local anesthetic infusion via a subfascially placed wound catheter did not improve pain relief when combined with basic analgesic regimen consisting of acetaminophen and NSAID with opioid used as rescue [60].
The limitations of this systematic review stem from the limitations of the included studies: particularly the inadequate study design (e.g., lack of double-blinding or explicit randomization) and lack of use of simple nonopioid analgesics when comparing more invasive techniques and a failure to evaluate all the potentially relevant analgesic agents and techniques for radical prostatectomy (especially infiltration techniques).

Thus, this review has identified several areas for future research when current data are insufficient or conflicting. There is a need for clinical trials evaluating multimodal analgesia techniques that would include combinations of paracetamol and NSAID/COX-2 selective inhibitor, and regional anesthetic techniques, with oral opioids administered only as rescue postoperatively. Future studies also need to evaluate the benefit to risk of continuous local anesthetic wound infusion and TAP blocks combined with multimodal analgesia. Also, large randomized clinical trials are necessary to assess the efficacy as well as optimal dose and duration of lidocaine intravenous infusion, ketamine and gabapentinoids. A study published after the deadline reported that a single preoperative dose $(900 \mathrm{mg})$ of gabapentin reduced pain scores but not opioid requirements [61].

Future trials should include multimodal enhanced rehabilitation protocols (fast track or enhanced recovery

Table 4 Summary of key results from included studies evaluating anesthetic interventions in patients undergoing radical

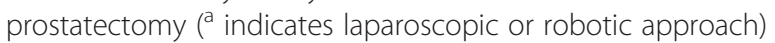

\begin{tabular}{|c|c|c|c|c|}
\hline Reference & Intervention studied & Pain scores & Supplementary analgesia & Time to first analgesic request \\
\hline Shir 1994 [37] & RA versus GA & $\downarrow$ at rest & $\downarrow \downarrow$ & - \\
\hline Haythornthwaite 1998 [25] & RA versus combined RA/ GA & NS & $\downarrow \downarrow$ & - \\
\hline Hong 2009 [45] & Combined RA/GA versus $G A^{a}$ & NS at rest, $\downarrow \downarrow$ on coughing & $\downarrow \downarrow$ & - \\
\hline Salonia 2004 [35] & SA versus $G A$ & $\downarrow$ at rest & - & - \\
\hline Salonia 2006 [36] & SA versus GA & $\downarrow \downarrow$ at rest & - & - \\
\hline
\end{tabular}

GA general anesthesia, RA regional anesthesia, SA spinal anesthesia, NS no significant difference between groups

- , not reported

$\downarrow$, decreased at a minority (50\% or less) of time points measured

$\downarrow \downarrow$, decreased at more than $50 \%$ of time points measured 
programs) as an integral part of the study design [62]. This will allow us to differentiate the effects of the analgesic interventions on perioperative outcome from those of the enhanced recovery programs that are becoming the standard of care. Also, there is a need for more procedurespecific studies comparing pain and analgesic requirements between open and minimal access (laparoscopic and robotic) surgical procedures.

\section{Conclusions}

This systematic review reveals that there is a lack of evidence to develop an optimal pain management protocol in patients undergoing radical prostatectomy. Most studies assessed unimodal analgesic approaches rather than a multimodal technique. There is a need for more procedure-specific studies comparing pain and analgesic requirements for open and minimally invasive surgical procedures. Finally, while we wait for appropriate procedure specific evidence from publication of adequate studies assessing optimal pain management after radical prostatectomy, we propose a basic analgesic guideline.

\section{Abbreviations}

PRISMA: Preferred reporting items for systematic reviews and meta-analyses; RCTs: Randomized controlled trials; NSAIDs: Non-steroidal anti-inflammatory drugs; COX-2: Cyclooxygenase-2; NMDA: N-methyl-D-aspartic acid; TAP: Transversus abdominis plane.

\section{Competing interests}

Over the past 5 years: Girish Joshi has received honoraria for consultancy and/or presentation from Pfizer, Mallinkrodt, and Pacira. Francis Bonnet, Narinder Rawal and Henrik Kehlet have received honoraria for consultancy and/or presentation from Pfizer. Francis Bonnet has received honorarium for consultancy and presentation from Nordic Pharma. Barrie Fischer has received honoraria for consultancy and/or presentation from Pfizer, B. Braun and AstraZeneca. Christian Simanski has received honoraria for consultancy and/or presentation from Gruenenthal, Merck, and Biomet. Edmund Neugebauer has received honoraria for consultancy and/or presentation from QRx Pharma, Gruenenthal, Janssen. The Anaesthesiology Unit of UWA, but not Stephan Schug privately, has received research and consultancy funding from Gruenenthal, CSL, Janssen Pharmaceuticals, Mundipharma, Pfizer, Phosphagenics and Biopharma.

\section{Authors' contributions}

Girish Joshi: Participated in conception and design of study, interpretation of data, and drafting and revising the manuscript. Thomas Jaschinski: Participated in the acquisition and analysis of data. Francis Bonnet: Participated in conception and design of study, interpretation of data, and drafting and revising the manuscript. Henrik Kehlet: Participated in conception and design of study, interpretation of data, and drafting and revising the manuscript. All authors read and approved the final manuscript.

\section{Acknowledgments \\ The authors would like to thank Esther Jacobs and Christoph Mosch, Cologne, Germany for assistance with data compilation. \\ All authors are members of the PROSPECT Working Group, which is supported by Pfizer Inc. New York, NY, USA. The PROSPECT Working Group members have been reimbursed by Pfizer Inc. for attending PROSPECT meetings to formulate the consensus recommendations. This paper makes no specific recommendations about the use of any medical products, drugs or equipment manufactured by Pfizer Inc. or by any of its subsidiaries.}

\section{PROSPECT collaboration}

Francis Bonnet, Department d' Anesthesie Reanimation, Hôspital Tenon, Paris, France.
H. Barrie J. Fischer, Department of Anaesthesiology, Alexandra Hospital, Redditch, Worcestershire, UK

Andrew Hill, Department of Surgery, University of Auckland, Auckland, New Zealand.

Girish P. Joshi, Department of Anesthesiology and Pain Management, University of Texas Southwestern Medical School, Dallas, TX, USA. Henrik Kehlet, Section for Surgical Pathophysiology, Rigshospitalet, Copenhagen University, Copenhagen, Denmark.

Philipp Lirk, Academic Medical Center, University of Amsterdam, The Netherlands.

Edmund A. M. Neugebauer, Institute of Research in Operative Medicine, University of Witten-Herdecke, Cologne, Germany.

Narinder Rawal, Department of Anaesthesia and Intensive Care, University Hospital, Örebro, Sweden.

Stephan A. Schug, School of Medicine and Pharmacology, University of Western Australia, Perth, Australia.

Christian J. P. Simanski, Department of Trauma and Orthopaedic Surgery Cologne-Merheim, University of Witten-Herdecke, Cologne, Germany. Marc Van de Velde, Department of Anesthesiology, Leuven, Netherlands. Marcel Vercauteren, Department of Anesthesiology, University of Antwerp, Antwerpen, Belgium.

\section{Author details}

'Department of Anesthesiology and Pain Management, University of Texas Southwestern Medical School, 5323 Harry Hines Blvd, Dallas, TX 75390-9068, USA. ${ }^{2}$ Institute for Research in Operative Medicine, Witten/Herdecke University, Cologne, Germany. ${ }^{3}$ Department d' Anesthesie Reanimation, Hôpital Tenon, Assistance Publique Hôpitaux de Paris Université Pierre \& Marie Curie, Paris, France. ${ }^{4}$ Section for Surgical Pathophysiology, Rigshospitalet, Copenhagen University, Copenhagen, Denmark.

Received: 21 August 2015 Accepted: 22 October 2015

Published online: 04 November 2015

\section{References}

1. Siegel R, Ward E, Brawley O, Jemal A. Cancer statistics, 2011: the impact of eliminating socioeconomic and racial disparities on premature cancer deaths. CA Cancer J Clin. 2011;61:212-36.

2. Parker C, Gillessen S, Heidenreich A, Horwich A. ESMO Guidelines Committee Cancer of the prostate: ESMO Clinical Practice Guidelines for diagnosis, treatment and follow-up. Ann Oncol 2015 Jul 22. [Epub ahead of print].

3. Liu JJ, Maxwell BG, Panousis P, Chung BI. Perioperative outcomes for laparoscopic and robotic compared with open prostatectomy using the National Surgical Quality Improvement Program (NSQIP) database. Urology. 2013;82:579-83.

4. Autorino R, Kaouk JH, Stolzenburg JU, Gill IS, Mottrie A, Tewari A, et al. Current status and future directions of robotic single-site surgery: a systematic review. Eur Urol. 2013;63:266-80.

5. Joshi GP, Schug S, Kehlet H. Procedure specific pain management and outcomes strategies. Best Prac Res Clin Anaesthesiol. 2014:28:191-201.

6. D'Alonzo RC, Gan TJ, Moul JW, Albala DM, Polascik TJ, Robertson CN, et al. A retrospective comparison of anesthetic management of robot-assisted laparoscopic radical prostatectomy versus radical retropubic prostatectomy. J Clin Anesth. 2009;21:322-8.

7. Woldu SL, Weinberg AC, Bergman A, Shapiro EY, Korets R, Motamedinia P, et al. Pain and analgesic use after robot-assisted radical prostatectomy. J Endourol. 2014:28:544-8.

8. Magheli A, Knoll N, Lein M, Hinz S, Kempkensteffen C, Gralla O. et al. Impact of fast-track postoperative care on intestinal function, pain, and length of hospital stay after laparoscopic radical prostatectomy. J Endourol. 2011;25:1143-7.

9. Moher D, Liberati A, Tetzlaff J, Altman DG. Reprint-preferred reporting items for systematic reviews and meta-analyses: the PRISMA statement. Phys Ther. 2009;89:873-80.

10. Higgins JPT, Altman DG, Sterne JAC. Chapter 8: Assessing risk of bias in included studies. In Higgins JPT, Green S (editors):Cochrane Handbook for Systematic Reviews of Interventions. Version 5.1.0 [updated March 2011]. The Cochrane Collaboration, 2011. Available from www.cochrane-handbook.org.

11. Allaire PH, Messick JM, Oesterling JE, Byer DE, Myers RP, Lieber MM, et al. A prospective randomized comparison of epidural infusion of fentanyl and intravenous administration of morphine by patient-controlled analgesia after radical retropubic prostatectomy. Mayo Clinic Proc. 1992;67:1031-41. 
12. Andrieu G, Roth B, Ousmane L, Castaner M, Petillot P, Vallet B, et al. The efficacy of intrathecal morphine with or without clonidine for postoperative analgesia after radical prostatectomy. Anesth Analg. 2009;108:1954-57.

13. Aribogan A, Doruk N, Aridogan A, Akin S, Balcioglu O. Patient-controlled epidural analgesia after major urologic surgeries. A comparison of tramado with or without bupivacaine. Urol Int. 2003;2:168-75.

14. Bilgin TE, Bozlu M, Atici S, Cayan S, Tasdelen B. Wound infiltration with bupivacaine and intramuscular diclofenac reduces postoperative tramadol consumption in patients undergoing radical retropubic prostatectomy: a prospective, double-blind, placebo-controlled, randomized study. Urology. 2011;78:1281-85

15. Borazan H, Tuncer S, Yalcin N, Erol A, Otelcioglu S. Effects of preoperative oral melatonin medication on postoperative analgesia, sleep quality, and sedation in patients undergoing elective prostatectomy: a randomized clinical trial. J Anesth. 2010;24:155-60.

16. Brown DR, Hofer RE, Patterson DE, Fronapfel PJ, Maxson PM, Narr BJ, et al. Intrathecal anesthesia and recovery from radical prostatectomy: a prospective, randomized, controlled trial. Anesthesiology. 2004;100:926-34

17. Chelly JE, Ploskanych T, Dai F, Nelson JB. Multimodal analgesic approach incorporating paravertebral blocks for open radical retropubic prostatectomy: a randomized double-blind placebo-controlled study. Can J Anaesth. 2011;58:371-8.

18. Fant F, Axelsson K, Sandblom D, Magnuson A, Andersson SO, Gupta A. Thoracic epidural analgesia or patient-controlled local analgesia for radical retropubic prostatectomy: a randomized, double-blind study. $\mathrm{Br} J$ Anaesth. 2011;107:782-9.

19. Gaitini L, Moskovitz B, Katz E, Vaisberg A, Vaida S, Nativ O. Sublingual buprenorphine compared to morphine delivered by a patient-controlled analgesia system as postoperative analgesia after prostatectomy. Urol Int. 1996:57:227-9.

20. Gottschalk A, Smith DS, Jobes DR, Kennedy SK, Lally SE, Noble VE, et al. Preemptive epidural analgesia and recovery from radical prostatectomy: a randomized controlled trial. JAMA. 1998;279:1076-82.

21. Groudine SB, Fisher HAG, Kaufman Jr RP, Patel MK, Wilkins LJ, Mehta SA, et al. Intravenous lidocaine speeds the return of bowel function, decreases postoperative pain, and shortens hospital stay in patients undergoing radical retropubic prostatectomy. Anesth Analg. 1998;86:235-9.

22. Gupta A, Fant F, Axelsson K, Sandblom D, Rykowski J, Johansson JE, et al. Postoperative analgesia after radical retropubic prostatectomy: a doubleblind comparison between low thoracic epidural and patient-controlled intravenous analgesia. Anesthesiology. 2006;105:784-93.

23. Habib AS, Polascik TJ, Weizer AZ, White WD, Moul JW, Elgasim MA, et al. Lidocaine patch for postoperative analgesia after radical retropubic prostatectomy. Anesth Analg. 2009;108:1950-3.

24. Habib AS, White WD, El Gasim MA, Saleh G, Polascik TJ, Moul JW, et al. Transdermal nicotine for analgesia after radical retropubic prostatectomy. Anesth Analg. 2008;107:999-1004

25. Haythornthwaite JA, Raja SN, Fisher B, Frank SM, Brendler SM, Shir Y. Pain and quality of life following radical retropubic prostatectomy. J Urol. 1999;160:1761-4.

26. Heid F, Schmidt-Glintzer A, Piepho T, Jage J. Epidural ropivacaine - Where are the benefits? A prospective, randomized, double-blind trial in patients with retropubic prostatectomy. Acta Anaesthesiol Scand. 2007:51:294-8.

27. Hohwu L, Akre O, Bergenwald L, Tornblom M, Gustafsson O. Oral oxycodone hydrochloride versus epidural anaesthesia for pain control after radical retropubic prostatectomy. Scand J Urol Nephrol. 2006;40:192-7.

28. Hong JY, Yang SC, Yi J, Kil HK. Epidural ropivacaine and sufentanil and the perioperative stress response after a radical retropubic prostatectomy. Acta Anaesthesiol Scand. 2011;55:282-9.

29. Huang JJ, Taguchi A, Hsu H, Andriole Jr GL. Preoperative oral rofecoxib does not decrease postoperative pain or morphine consumption in patients after radical prostatectomy: a prospective, randomized, double-blinded, placebo-controlled trial. J Clin Anesth. 2001;13:94-7.

30. Katz J, Schmid R, Snijdelaar DG, Coderre TJ, McCartney CJL, Wowk A. Pre-emptive analgesia using intravenous fentanyl plus low-dose ketamine for radical prostatectomy under general anesthesia does not produce short-term or long-term reductions in pain or analgesic use. Pain. 2004;110:707-18.

31. Liu S, Carpenter RL, Mulroy MF, Weissman RM, McGill TJ, Rupp SM, et al. Intravenous versus epidural administration of hydromorphone: effects on analgesia and recovery after radical retropubic prostatectomy. Anesthesiology. 1995;82:682-8.
32. Mayson KV, Gofton EA, Chambers KG. Premedication with low dose oral clonidine does not enhance postoperative analgesia of intrathecal morphine. Can J Anesth. 2000:47:752-7.

33. Mazaris EM, Varkarakis I, Chrisofos M, Skolarikos A, loannidis K, Dellis A, et al Use of Nonsteroidal anti-inflammatory drugs after radical retropubic prostatectomy: a prospective, randomized trial. Urology. 2008;72:1293-7.

34. Ormiston MC, Vaughton KC, Thornton EJ. The comparative effectiveness of tiaprofenic acid and aspirin in the treatment of post-prostatectomy pain. $\mathrm{Br}$ J Clin Pract. 1981;35:360-2.

35. Salonia A, Crescenti A, Suardi N, Memmo A, Naspro R, Bocciardi AM, et al. General versus spinal anesthesia in patients undergoing radical retropubic prostatectomy: results of a prospective, randomized study. Urology. 2004;64:95-100.

36. Salonia A, Suardi N, Crescenti A, Colombo R, Rigatti P, Montorsi F. General versus spinal anesthesia with different forms of sedation in patients undergoing radical retropubic prostatectomy: results of a prospective, randomized study. Int J Urol. 2006;13:1185-90.

37. Shir Y, Raja SN, Frank SM. The effect of epidural versus general anesthesia on postoperative pain and analgesic requirements in patients undergoing radical prostatectomy. Anesthesiology. 1994;80:49-56.

38. Snijdelaar DG, Cornelisse HB, Schmid RL, Katz J. A randomised, controlled study of peri-operative low dose $s(+)$-ketamine in combination with postoperative patient-controlled $s(+)$-ketamine and morphine after radical prostatectomy. Anaesthesia. 2004;59:222-8.

39. Snijdelaar DG, Koren G, Katz J. Effects of perioperative oral amantadine on postoperative pain and morphine consumption in patients after radical prostatectomy: results of a preliminary study. Anesthesiology. 2004;100:134-41.

40. Tauzin-Fin P, Sesay M, Delort-Laval S, Krol-Houdek MC, Maurette P. Intravenous magnesium sulphate decreases postoperative tramadol requirement after radical prostatectomy. Eur J Anaesthesiol. 2006;23:1055-59.

41. Tauzin-Fin P, Sesay M, Svartz L, Krol-Houdek MC, Maurette P. Sublingual oxybutynin reduces postoperative pain related to indwelling bladder catheter after radical retropubic prostatectomy. Br J Anaesth. 2007;99:572-5.

42. Tauzin-Fin P, Sesay M, Svartz L, Krol-Houdek MC, Maurette P. Wound infiltration with magnesium sulphate and ropivacaine mixture reduces postoperative tramadol requirements after radical prostatectomy. Acta Anaesthesiol Scand. 2009:53:464-9.

43. Wu CL, Partin AW, Rowlingson AJ, Kalish MA, Walsh PC, Fleisher LA. Efficacy of continuous local anesthetic infusion for postoperative pain after radical retropubic prostatectomy. Urology. 2005;66:366-70.

44. Lauwick S, Kim DJ, Mistraletti G, Carli F. Functional walking capacity as an outcome measure of laparoscopic prostatectomy: the effect of lidocaine infusion. Br J Anaesth. 2009;103:213-9.

45. Hong JY, Lee SJ, Rha KH, Roh GU, Kwon SY, Kil HK. Effects of thoracic epidural analgesia combined with general anesthesia on intraoperative ventilation/oxygenation and postoperative pulmonary complications in robotassisted laparoscopic radical prostatectomy. J Endourol. 2009;23:1843-9.

46. Lee C, Song YK, Jeong HM, Park SN. The effects of magnesium sulfate infiltration on perioperative opioid consumption and opioid-induced hyperalgesia in patients undergoing robot-assisted laparoscopic prostatectomy with remifentanil-based anesthesia. Korean J Anesthesiol. 2011;61:244-50

47. Lukasewycz S, Holman M, Kozlowski P, Porter CR, Odom E, Bernands C, et al. Does a perioperative belladonna and opium suppository improve postoperative pain following robotic assisted laparoscopic radical prostatectomy? Results of a single institution randomized study. Can J Urol. 2010;17:5377-82.

48. Larijani GE, Goldberg ME, Gratz I, Warshal DP. Analgesic and hemodynamic effects of a single 7.5-mg intravenous dose of morphine in patients with moderate-to-severe postoperative pain. Pharmacotherapy. 2004:24:1675-80.

49. Weinberg AC, Woldu SL, Bergman A, Roychoudhury A, Patel T, Berg W, et al. Dorsal penile nerve block for robot-assisted radical prostatectomy catheter related pain: a randomized, double-blind, placebo-controlled trial. Springerplus. 2014;3:181.

50. Fuller A, Vanderhaeghe L, Nott L, Martin PR, Pautler SE. Intravesical ropivacaine as a novel means of analgesia post-robot-assisted radical prostatectomy: a randomized, double-blind, placebo-controlled trial. J Endourol. 2013;27:313-7.

51. Gardner TA, Bissonette EA, Petroni GR, McClain R, Sokoloff MH, Theodorescu D. Surgical and postoperative factors affecting length of hospital stay after radical prostatectomy. Cancer. 2000;89:424-30 
52. Wheeler M, Oderda GM, Ashburn MA, Lipman AG. Adverse events associated with postoperative opioid analgesia: a systematic review. J Pain. 2002;3:159-80

53. Hegi TR, Bombeli T, Seifert B, Baumann PC, Haller U, Zalunardo MP, et al. Effect of rofecoxib on platelet aggregation and blood loss in gynaecological and breast surgery compared with diclofenac. Br J Anaesth. 2004;92:523-31.

54. Dirkmann D, Groeben H, Farhan H, Stahl SL, Eikermann M. Effects of parecoxib on analgesia benefits and blood loss following open prostatectomy: a multicentre randomized trial. BMC Anesthesiol. 2015;15:31.

55. Nuri Deniz M, Erhan E, Ugur G. Intrathecal morphine reduces postoperative tramadol consumption in patients undergoing radical retropubic prostatectomy: a randomized trial. Eur Rev Med Pharmacol Sci. 2013;17:834-8.

56. Ozbek H, Deniz MN, Erakgun A, Erhan E. Comparison of 75 and $150 \mu \mathrm{g}$ doses of intrathecal morphine for postoperative analgesia after transurethral resection of the prostate under spinal anesthesia. J Opioid Manag. 2013;9:415-20.

57. Mir MC, Joseph B, Zha R, Bolton DM, Gyomber D, Lawrentschuk N Effectiveness of epidural versus alternate analgesia for pain relief after radical prostatectomy and correlation with biochemical recurrence in men with prostate cancer. Res Rep Urol. 2013;5:139-45.

58. Elkassabany N, Ahmed M, Malkowicz SB, Heitjan DF, Isserman JA, Ochroch EA. Comparison between the analgesic efficacy of transversus abdominis plane (TAP) block and placebo in open retropubic radical prostatectomy: a prospective, randomized, double-blinded study. J Clin Anesth. 2013;25:459-65.

59. Skjelsager A, Ruhnau B, Kistorp TK, Kridina I, Hvarness H, Mathiesen O, et al. Transversus abdominis plane block or subcutaneous wound infiltration after open radical prostatectomy: a randomized study. Acta Anaesthesiol Scand. 2013:57:502-8.

60. Kristensen BS, Fenger-Eriksen C, Pedersen KV, Felsby S. Wound infusion of bupivacaine following radical retropubic prostatectomy: a randomized placebo-controlled clinical study. Acta Anaesthesiol Scand. 2013;30:124-8.

61. Deniz MN, Sertoz N, Erhan E, Ugur G. Effects of preoperative gabapentin on postoperative pain after radical retropubic prostatectomy. J Int Med Res. 2012;40:2362-9.

62. Santa Mina D, Matthew AG, Hilton WJ, Au D, Awasthi R, Alibhai SMH, et al. Prehabilitation for men undergoing radical prostatectomy: a multicentre, pilot randomized controlled trial. BMC Surg. 2014;14:89.

\section{Submit your next manuscript to BioMed Central and take full advantage of:}

- Convenient online submission

- Thorough peer review

- No space constraints or color figure charges

- Immediate publication on acceptance

- Inclusion in PubMed, CAS, Scopus and Google Scholar

- Research which is freely available for redistribution

Submit your manuscript at www.biomedcentral.com/submit 